\title{
Cortical proprioceptive processing is altered in children with diplegic cerebral palsy
}

\section{Harri Piitulainen, Timo Nurmi, Jaakko Vallinoja, Julia Jaatela, Elina Ylitalo, Helena Mäenpää}

${ }^{1}$ Faculty of Sport and Health Sciences, University of Jyväskylä, Jyväskylä, Finland

${ }^{2}$ Motion Analysis Laboratory, Helsinki University Hospital (HUH) Children and Adolescents, Helsinki, Finland

${ }^{3}$ Department of Neuroscience and Biomedical Engineering, Aalto University School of Science, Espoo, Finland

\section{Introduction}

Children with cerebral palsy (CP) have various motor impairments, but less is known about the processing deficits of the somatosensory afference, especially in the proprioceptive domain.

Proprioception ("the movement sense") is behaviorally impaired primarily in the more affected hand of children with $\mathrm{CP}^{1,2}$ and bilaterally in the lower limbs ${ }^{2}$. There are no prior studies quantifying the cortical proprioceptive processing in $\mathrm{CP}$, but hand representation area is diminished to tactile stimuli in hemiplegic $\mathrm{CP}^{3}$ and the primary cortical response to tibial nerve stimulation is reduced in diplegic $\mathrm{CP}^{4}$.

\section{Research Question}

We examined whether the cortical proprioceptive processing is altered in children with hemiplegic (HP) or diplegic (DP) CP compared to typically developed (TD) controls, using passive-movement stimuli of the limbs in magnetoencephalography (MEG), and whether the response strengths are associated with behavioral motor function (Box and blocks, 9-hole-peg and standing-postural balance tests).

\section{Methods}

We extracted strength of cortical proprioceptive processing in $15 \mathrm{HP}$ (13.3 \pm 2.2 years), $11 \mathrm{DP}(13.3 \pm 2.2$ years) and 32 TD controls ( $14 \pm 2.4$ years) using proprioceptive stimulation ( 60 stimulus/limb) of passive right and left index-finger movements and ankle-joint rotations evoked by pneumaticmovement actuators ${ }^{5,6}$ during 306-channel MEG (Elekta Neuromag ${ }^{\text {TM }}$, Finland) recordings. The proprioceptive responses were averaged across stimuli, and the response strength was quantified as the strongest primary response peak in the vector sum of a gradiometer pair showing the peak response over the hand or foot area of the primary somatosensory cortex.

\section{Results}

The cortical proprioceptive response for the non-dominant hand was weaker for DP $(57.9 \pm 18.2 \mathrm{fT} / \mathrm{cm})$ than TD group (80.1 $\pm 23.6 \mathrm{fT} / \mathrm{cm}, p=0.019)$, but did not differ from HP $(71.4 \pm 39.5 \mathrm{fT} / \mathrm{cm}, \mathrm{p}=0.475)$. There were no other significant differences for the hand responses, nor foot responses $(p=0.125-$ 0.894).

The more affected (non-dominant) hand in DP group showed weaker proprioceptive responses than their less affected (dominant) hand $(p=0.017)$. No other between limb differences were observed.

The sensorimotor function was worse in all tests for children with $\mathrm{CP}(\mathrm{p}<0.001-0.05)$, with no differences between HP and DP groups. The better hand-gross-motor function (Box and blocks test) of 
the non- and dominant hands were associated with stronger corresponding hand proprioceptive response ( $r=0.30, p=0.037$ and $r=0.28, p=0.049$ respectively) across all participants. However, this correlation was not detected in the sub-groups.

\section{Discussion}

Cortical proprioceptive processing is diminished for the more affected hand in diplegic CP in accordance with previous observations on other somatosensory modalities ${ }^{1,2}$. The MEG response strengths were only weakly linked to motor performance and should thus be confirmed with larger sample sizes and more robust measures such as corticokinematic coherence ${ }^{7}$.
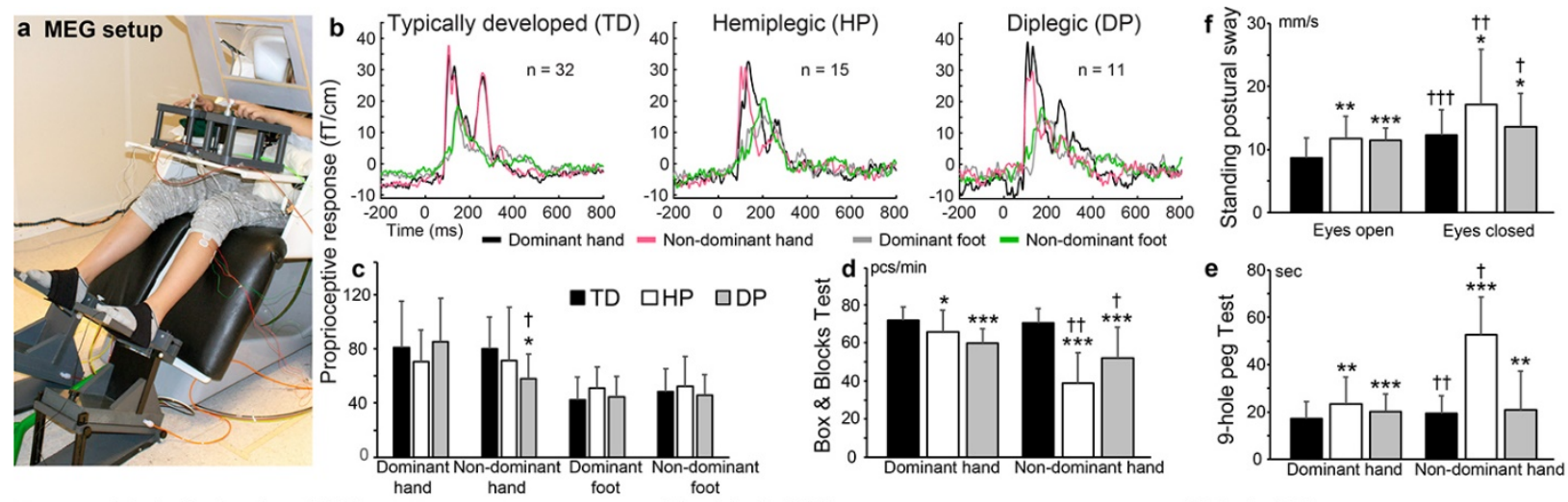

g Typically developed (TD)
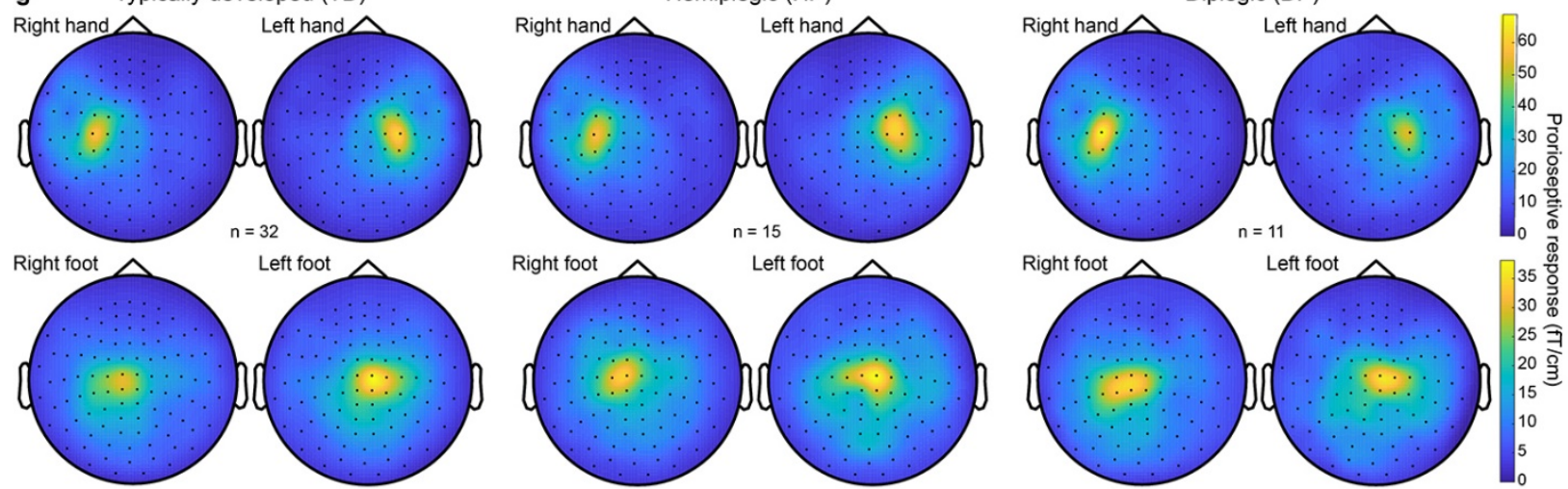

Figure 1. (a) experimental setup. (b and c) group-means for propriocetive MEG response time courses and peak values. (d) gross-motor test. (e) fine-motor test. (f) standing balance test feet together. (g) group-mean MEG topographic distributions of the prorpioceptive responses. Note that the distributions are for right and left limbs, not according to limb dominance. ${ }^{\star},{ }^{\star \star},{ }^{\star \star \star}=$ diffrent from TD group at $p<0.05, p<0.01, p<0.001 . \dagger, \dagger \dagger, \dagger+\dagger=$ different from dominant hand or eyes open standing at $p<0.05, p<0.01, p<0.001$

\section{Keywords}

CEREBRAL PALSY; PROPRIOCEPTION; SOMATOSENSORY; CORTEX; MOTOR FUNCTION; THE BRAIN

\section{Disclosure statement}

This abstract is financially supported by grants from the Academy of Finland (grants \#296240, \#326988, \#307250 and \#327288) Jane and Aatos Erkko Foundation to Harri Piitulainen. We thank technical support from Helge Kainulainen in building the pneumatic-movement actuators at Aalto Neurolmaging, Aalto University, Espoo, Finland. 


\section{References}

1. Goble TJ et al. 2009. Deficits in the ability to use proprioceptive feedback in children with hemiplegic cerebral palsy. Int J Rehabil Res 32:267-9

2. Wingert JT et al. 2009. Joint-Position Sense and Kinesthesia in Cerebral Palsy. Arch Phys Med Rehabil 90:447-53

3. Nevalainen P et al. 2012 Dev Med Child Neurol 54:361-7

4. Kurz MJ et al. 2011. Neuromagnetic activity in the somatosensory cortices of children with cerebral palsy. Neurosci Lett 490:1-5

5. Piitulainen et al. 2015. MEG-compatible pneumatic stimulator to elicit passive finger and toe movements. Neuroimage 2015 112:310-7.

6. Piitulainen et al. 2018. Cortical Proprioceptive Processing Is Altered by Aging. Front Aging Neurosci 10:147

7. Piitulainen et al. 2013. Corticokinematic coherence during active and passive finger movements. Neuroscience 238:361-70 